\title{
An upper bound for the regularity of symbolic powers of edge ideals of chordal graphs
}

\author{
Seyed Amin Seyed Fakhari* \\ School of Mathematics, Statistics and Computer Science \\ College of Science, University of Tehran \\ Tehran, Iran. \\ Institute of Mathematics \\ Vietnam Academy of Science and Technology \\ 18 Hoang Quoc Viet, Hanoi, Vietnam. \\ aminfakhari@ut.ac.ir
}

Submitted: Mar 9, 2019; Accepted: Mar 31, 2019; Published: Apr 19, 2019

(C) The author. Released under the CC BY-ND license (International 4.0).

\begin{abstract}
Assume that $G$ is a chordal graph with edge ideal $I(G)$ and ordered matching number ord-match $(G)$. For every integer $s \geqslant 1$, we denote the $s$-th symbolic power of $I(G)$ by $I(G)^{(s)}$. It is shown that $\operatorname{reg}\left(I(G)^{(s)}\right) \leqslant 2 s+\operatorname{ord-match}(G)-1$. As a consequence, we determine the regularity of symbolic powers of edge ideals of chordal Cameron-Walker graphs.
\end{abstract}

Mathematics Subject Classifications: 13D02, 05E99

\section{Introduction}

Let $\mathbb{K}$ be a field and $S=\mathbb{K}\left[x_{1}, \ldots, x_{n}\right]$ be the polynomial ring in $n$ variables over $\mathbb{K}$. Suppose that $M$ is a nonzero graded $S$-module with minimal free resolution

$$
0 \longrightarrow \cdots \longrightarrow \bigoplus_{j} S(-j)^{\beta_{1, j}(M)} \longrightarrow \bigoplus_{j} S(-j)^{\beta_{0, j}(M)} \longrightarrow M \longrightarrow 0
$$

The Castelnuovo-Mumford regularity (or simply, regularity) of $M$, denoted by $\operatorname{reg}_{S}(M)$, is defined as

$$
\operatorname{reg}_{S}(M)=\max \left\{j-i \mid \beta_{i, j}(M) \neq 0\right\} .
$$

*This research is partially funded by the Simons Foundation Grant Targeted for Institute of Mathematics, Vietnam Academy of Science and Technology. 
As a convention, we set $\operatorname{reg}_{S}(M)=\infty$, when $M$ is the zero module. When there is no fear of confusion, we delete the subscript $S$ in the above notation. We mention that the Castelnuovo-Mumford regularity is an important invariant in commutative algebra and algebraic geometry.

There is a natural correspondence between quadratic squarefree monomial ideals of $S$ and finite simple graphs with $n$ vertices. To every simple graph $G$ with vertex set $V(G)=\left\{x_{1}, \ldots, x_{n}\right\}$ and edge set $E(G)$, we associate its edge ideal $I=I(G)$ defined by

$$
I(G)=\left(x_{i} x_{j}: x_{i} x_{j} \in E(G)\right) \subseteq S .
$$

Computing and finding bounds for the regularity of edge ideals and their powers have been studied by a number of researchers (see for example [1], [2], [3], [4], [5], [8], [10], [15], [16], [17], [18], [20] and [22]).

Katzman [16], proved that for any graph $G$,

$$
\operatorname{reg}(I(G)) \geqslant \operatorname{ind}-\operatorname{match}(G)+1,
$$

where ind-match $(G)$ denotes the induced matching number of $G$. Beyarslan, Hà and Trung [5], generalized Katzman's inequality by showing that

$$
\operatorname{reg}\left(I(G)^{s}\right) \geqslant 2 s+\text { ind-match }(G)-1,
$$

for every integer $s \geqslant 1$. Recently, Gu, Hà, O'Rourke and Skelton [9] proved the same inequality for symbolic powers. More explicit, they proved that

$$
\operatorname{reg}\left(I(G)^{(s)}\right) \geqslant 2 s+\text { ind-match }(G)-1,
$$

for any graph $G$ and any integer $s \geqslant 1$.

Our approach is to determine an upper bound for the regularity of symbolic powers of edge ideals. Indeed, Hà and Van Tuyl [11, Theorem 6.7] proved that for every graph $G$,

$$
\operatorname{reg}(I(G)) \leqslant \operatorname{match}(G)+1,
$$

where match $(G)$ denotes the matching number of $G$. This inequality was strengthen by Constantinescu and Varbaro [7, Remark 4.8] (see also [19, Corollary 2.5]). To be more precise, let $G$ be a graph with ordered matching number ord-match $(G)$ (see Definition 1). Then

$$
\operatorname{reg}(I(G)) \leqslant \operatorname{ord}-\operatorname{match}(G)+1 .
$$

It is natural to ask whether the inequalities

$$
\operatorname{reg}\left(I(G)^{s}\right) \leqslant 2 s+\operatorname{ord}-\operatorname{match}(G)-1,
$$

and

$$
\operatorname{reg}\left(I(G)^{(s)}\right) \leqslant 2 s+\operatorname{ord}-\operatorname{match}(G)-1
$$

are true. To the best of our knowledge, it is not known whether inequality (1) is true. In this paper, we investigate inequality (2) and as the main result, we show that inequality (2) holds for any chordal graph $G$ and for any integer $s \geqslant 1$ (see Theorem 3. As a consequence, we determine the regularity of symbolic powers of edge ideals of graphs belonging to the following classes. 
1. Chordal Cameron-Walker graphs.

2. Complete graphs $K_{n}$.

3. $K_{n}-e$, where $e$ is an arbitrary edge of $K_{n}$.

\section{Preliminaries}

In this section, we provide the definitions and basic facts which will be used in the next section.

Let $G$ be a simple graph with vertex set $V(G)=\left\{x_{1}, \ldots, x_{n}\right\}$ and edge set $E(G)$. For a vertex $x_{i}$, the neighbor set of $x_{i}$ is $N_{G}\left(x_{i}\right)=\left\{x_{j} \mid x_{i} x_{j} \in E(G)\right\}$ and we set $N_{G}\left[x_{i}\right]=N_{G}\left(x_{i}\right) \cup\left\{x_{i}\right\}$. The cardinality of $N_{G}\left(x_{i}\right)$ is called the degree of $x_{i}$. A vertex of degree one is a leaf and the unique edge incident to a leaf is called a pendant edge. A pendant triangle of $G$ is a triangle $T$ of $G$, with the property that exactly two vertices of $T$ have degree two in $G$. For every subset $U \subset V(G)$, the graph $G-U$ has vertex set $V(G-U)=V(G) \backslash U$ and edge set $E(G-U)=\{e \in E(G) \mid e \cap U=\varnothing\}$. Also, for any edge $e \in E(G)$, we done by $G-e$, the graph obtained from $G$ by deleting the edge $e$. A subgraph $H$ of $G$ is called induced provided that two vertices of $H$ are adjacent if and only if they are adjacent in $G$. The induced subgraph of $G$ on the vertex set $U \subseteq V(G)$ will be denoted by $G_{U}$. A graph $G$ is called chordal if it has no induced cycle of length at least four. A subset $W$ of $V(G)$ is a clique of $G$ if every two distinct vertices of $W$ are adjacent in $G$. A vertex $x$ of $G$ is a simplicial vertex if $N_{G}(x)$ is a clique. It is well-known that every chordal graph has a simplicial vertex. A subset $A$ of $V(G)$ is called an independent subset of $G$ if there are no edges among the vertices of $A$. A subset $C$ of $V(G)$ is a vertex cover of $G$ if every edge of $G$ is incident to at least one vertex of $C$. A vertex cover $C$ is a minimal vertex cover if no proper subset of $C$ is a vertex cover of $G$. The set of minimal vertex covers of $G$ will be denoted by $\mathcal{C}(G)$.

For every subset $C$ of $\left\{x_{1}, \ldots, x_{n}\right\}$, we denote by $\mathfrak{p}_{C}$, the monomial prime ideal which is generated by the variables belong to $C$. It is well-known that for every graph $G$ with edge ideal $I(G)$,

$$
I(G)=\bigcap_{C \in \mathcal{C}(G)} \mathfrak{p}_{C}
$$

Let $G$ be a graph. A subset $M \subseteq E(G)$ is a matching if $e \cap e^{\prime}=\varnothing$, for every pair of edges $e, e^{\prime} \in M$. The cardinality of the largest matching of $G$ is called the matching number of $G$ and is denoted by match $(G)$. A matching $M$ of $G$ is an induced matching of $G$ if for every pair of edges $e, e^{\prime} \in M$, there is no edge $f \in E(G) \backslash M$ with $f \subset e \cup e^{\prime}$. The cardinality of the largest induced matching of $G$ is the induced matching number of $G$ and is denoted by ind-match $(G)$.

Definition 1. Let $G$ be a graph, and let $M=\left\{\left\{a_{i}, b_{i}\right\} \mid 1 \leqslant i \leqslant r\right\}$ be a nonempty matching of $G$. We say that $M$ is an ordered matching of $G$ if the following hold:

(1) $A:=\left\{a_{1}, \ldots, a_{r}\right\} \subseteq V(G)$ is a set of independent vertices of $G$; and 
(2) $\left\{a_{i}, b_{j}\right\} \in E(G)$ implies that $i \leqslant j$.

The ordered matching number of $G$, denoted by $\operatorname{ord}-\operatorname{match}(G)$, is defined to be

$$
\operatorname{ord}-\operatorname{match}(G)=\max \{|M| \mid M \subseteq E(G) \text { is an ordered matching of } G\} .
$$

We close this section by recalling the definition of symbolic powers.

Let $I$ be an ideal of $S$ and let $\operatorname{Min}(I)$ denote the set of minimal primes of $I$. For every integer $s \geqslant 1$, the $s$-th symbolic power of $I$, denoted by $I^{(s)}$, is defined to be

$$
I^{(s)}=\bigcap_{\mathfrak{p} \in \operatorname{Min}(I)} \operatorname{Ker}\left(R \rightarrow\left(R / I^{s}\right)_{\mathfrak{p}}\right) .
$$

Let $I$ be a squarefree monomial ideal in $S$ and suppose that $I$ has the irredundant primary decomposition

$$
I=\mathfrak{p}_{1} \cap \ldots \cap \mathfrak{p}_{r},
$$

where every $\mathfrak{p}_{i}$ is an ideal generated by a subset of the variables of $S$. It follows from $[12$, Proposition 1.4.4] that for every integer $s \geqslant 1$,

$$
I^{(s)}=\mathfrak{p}_{1}^{s} \cap \ldots \cap \mathfrak{p}_{r}^{s} .
$$

We set $I^{(s)}=S$, for any integer $s \leqslant 0$.

\section{Main results}

In this section, we prove the main result of this paper, Theorem 3. The proof is based on an inductive argument and the following lemma has a crucial role in our induction.

Lemma 2. Let $G$ be a graph and assume that $x_{1}$ is a simplicial vertex of $G$, with $N_{G}\left(x_{1}\right)=$ $\left\{x_{2}, \ldots x_{d}\right\}$, for some integer $d \geqslant 1$. Then for every integer $s \geqslant 1$,

$$
\left(I(G)^{(s)}: x_{1} x_{2} \ldots x_{d}\right)=I(G)^{(s-d+1)} .
$$

Proof. Let $C$ be a minimal vertex cover of $G$. Since $G_{N_{G}\left[x_{1}\right]}$ is a complete graph, it follows that $\left|C \cap N_{G}\left[x_{1}\right]\right| \geqslant d-1$. On the other hand, $N_{G}\left[x_{1}\right] \nsubseteq C$, as otherwise $C \backslash\left\{x_{1}\right\}$ would be vertex cover of $G$ which is strictly contained in $C$. Thus, $\left|C \cap N_{G}\left[x_{1}\right]\right|=d-1$. Consequently,

$$
\left(\mathfrak{p}_{C}^{s}: x_{1} x_{2} \ldots x_{d}\right)=\mathfrak{p}_{C}^{s-d+1}
$$

We know that $I(G)=\bigcap_{C \in \mathcal{C}(G)} \mathfrak{p}_{C}$. Thus,

$$
I(G)^{(s)}=\bigcap_{C \in \mathcal{C}(G)} \mathfrak{p}_{C}^{s}
$$

It follows that

$$
\left(I(G)^{(s)}: x_{1} x_{2} \ldots x_{d}\right)=\bigcap_{C \in \mathcal{C}(G)}\left(\mathfrak{p}_{C}^{s}: x_{1} x_{2} \ldots x_{d}\right)=\bigcap_{C \in \mathcal{C}(G)} \mathfrak{p}_{C}^{s-d+1}=I(G)^{(s-d+1)} .
$$


We are now ready to prove our main result.

Theorem 3. Let $G$ be a chordal graph. Then for every integer $s \geqslant 1$, we have

$$
\operatorname{reg}\left(I(G)^{(s)}\right) \leqslant 2 s+\operatorname{ord}-\operatorname{match}(G)-1 .
$$

Proof. Suppose $V(G)=\left\{x_{1}, \ldots, x_{n}\right\}$. We use induction on $n+s$. The assertion follows from [11, Corollary 6.9], for $s=1$. Thus, assume that $s \geqslant 2$. If $n=2$, then $I(G)=\left(x_{1} x_{2}\right)$. Consequently, $I(G)^{(s)}=\left(x_{1}^{s} x_{2}^{s}\right)$ and

$$
\operatorname{reg}\left(I(G)^{(s)}\right)=2 s=2 s+\operatorname{ord}-\operatorname{match}(G)-1 .
$$

Hence, suppose $n \geqslant 3$.

Assume without loss of generality that $x_{1}$ is a simplicial vertex of $G$ and $N_{G}\left(x_{1}\right)=$ $\left\{x_{2}, \ldots, x_{d}\right\}$, for some integer $d \geqslant 2$. Let $S_{1}=\mathbb{K}\left[x_{2}, \ldots, x_{n}\right]$ be the polynomial ring obtained from $S$ by deleting the variable $x_{1}$ and consider the ideals $I_{1}=I(G)^{(s)} \cap S_{1}$ and $I_{1}^{\prime}=\left(I(G)^{(s)}: x_{1}\right)$. It follows from $[8$, Lemma 2.10] that

$$
\operatorname{reg}\left(S / I(G)^{(s)}\right) \leqslant \max \left\{\operatorname{reg}_{S_{1}}\left(S_{1} / I_{1}\right), \operatorname{reg}_{S}\left(S / I_{1}^{\prime}\right)+1\right\}
$$

Notice that

$$
I_{1}=\left(I(G) \cap S_{1}\right)^{(s)}=I\left(G \backslash x_{1}\right)^{(s)} .
$$

As $G \backslash x_{1}$ is a chordal graph, it follows from the induction hypothesis that

$$
\operatorname{reg}_{S_{1}}\left(S_{1} / I_{1}\right) \leqslant 2 s+\operatorname{ord}-\operatorname{match}\left(G \backslash x_{1}\right)-2 \leqslant 2 s+\operatorname{ord}-\operatorname{match}(G)-2 .
$$

Thus, using the inequality (3), it is enough to prove that

$$
\operatorname{reg}\left(S / I_{1}^{\prime}\right) \leqslant 2 s+\operatorname{ord}-\operatorname{match}(G)-3 .
$$

For every subset $A \subseteq V(G)$, set $\mathrm{x}_{A}:=\prod_{x_{i} \in A} x_{i}$.

Claim. For every subset $W \subseteq N_{G}\left[x_{1}\right]$, with $x_{1} \in W$, we have

$$
\operatorname{reg}\left(S / I_{1}^{\prime}\right) \leqslant \max \left\{\operatorname{reg}\left(\frac{S}{I(G \backslash A)^{(s)}: \mathrm{x}_{B}}\right)+|B|-1 \mid x_{1} \in B, A \cap B=\varnothing, A \cup B=W\right\} .
$$

Proof of the Claim. Without loss of generality, assume that $W=\left\{x_{1}, \ldots, x_{t}\right\}$, for some integer $1 \leqslant t \leqslant d$. We use induction on $t$. There is nothing to prove for $t=1$. Therefore, suppose that $t \geqslant 2$. Set $W^{\prime}=\left\{x_{1}, \ldots, x_{t-1}\right\}$. We know from the induction hypothesis that

$$
\operatorname{reg}\left(S / I_{1}^{\prime}\right) \leqslant \max \left\{\operatorname{reg}\left(\frac{S}{I(G \backslash A)^{(s)}: \mathrm{x}_{B}}\right)+|B|-1 \mid x_{1} \in B, A \cap B=\varnothing, A \cup B=W^{\prime}\right\} .
$$


For every pair of subsets $A, B \subseteq V(G)$ with $x_{1} \in B, A \cap B=\varnothing$ and $A \cup B=W^{\prime}$, it follows from [8, Lemma 2.10] that

$$
\begin{aligned}
& \operatorname{reg}\left(\frac{S}{I(G \backslash A)^{(s)}: \mathrm{x}_{B}}\right)+|B|-1 \\
& \leqslant \max \left\{\operatorname{reg}\left(\frac{S}{\left(I(G \backslash A)^{(s)}: \mathrm{x}_{B}\right), x_{t}}\right)+|B|-1, \operatorname{reg}\left(\frac{S}{I(G \backslash A)^{(s)}: x_{t} \mathrm{x}_{B}}\right)+|B|\right\} \\
& =\max \left\{\operatorname{reg}\left(\frac{S}{\left(I(G \backslash A)^{(s)}, x_{t}\right): \mathrm{x}_{B}}\right)+|B|-1, \operatorname{reg}\left(\frac{S}{I(G \backslash A)^{(s)}: x_{t} \mathrm{x}_{B}}\right)+|B|\right\} \\
& =\max \left\{\operatorname{reg}\left(\frac{S}{I\left(G \backslash\left(A \cup\left\{x_{t}\right\}\right)\right)^{(s)}: \mathrm{x}_{B}}\right)+|B|-1, \operatorname{reg}\left(\frac{S}{I(G \backslash A)^{(s)}: \mathrm{x}_{B \cup\left\{x_{t}\right\}}}\right)+|B|\right\} .
\end{aligned}
$$

The claim now follows by combining this inequality and inequality (4).

Substituting $W=N_{G}\left[x_{1}\right]$ in the claim, we deduce that

$$
\operatorname{reg}\left(S / I_{1}^{\prime}\right) \leqslant \max \left\{\operatorname{reg}\left(\frac{S}{I(G \backslash A)^{(s)}: \mathrm{x}_{B}}\right)+|B|-1 \mid x_{1} \in B, A \cap B=\varnothing, A \cup B=N_{G}\left[x_{1}\right]\right\}
$$

Let $A$ and $B$ be subsets of $V(G)$ with $x_{1} \in B, A \cap B=\varnothing$ and $A \cup B=N_{G}\left[x_{1}\right]$. Obviously, $G \backslash A$ is a chordal graph and $x_{1}$ is a simplicial vertex of this graph. Clearly, $N_{G \backslash A}\left[x_{1}\right]=B$. Hence, using Lemma 2, we conclude that

$$
\left(I(G \backslash A)^{(s)}: \mathrm{x}_{B}\right)=I(G \backslash A)^{(s-|B|+1)} .
$$

We consider the following cases.

Case 1. If $|B| \geqslant s+1$, then $I(G \backslash A)^{(s-|B|+1)}=S$. Thus,

$$
\operatorname{reg}\left(\frac{S}{I(G \backslash A)^{(s)}: \mathrm{x}_{B}}\right)+|B|-1=-\infty<2 s+\operatorname{ord}-\operatorname{match}(G)-3 .
$$

Case 2. If $2 \leqslant|B| \leqslant s$, then it follows from the induction hypothesis that

$$
\begin{aligned}
& \operatorname{reg}\left(\frac{S}{I(G \backslash A)^{(s-|B|+1)}}\right) \leqslant 2(s-|B|+1)+\operatorname{ord}-\operatorname{match}(G \backslash A)-2 \\
& \leqslant 2(s-|B|+1)+\text { ord-match }(G)-2 .
\end{aligned}
$$

Therefore,

$$
\begin{aligned}
& \operatorname{reg}\left(\frac{S}{I(G \backslash A)^{(s)}: \mathrm{x}_{B}}\right)+|B|-1=\operatorname{reg}\left(\frac{S}{I(G \backslash A)^{(s-|B|+1)}}\right)+|B|-1 \\
& \leqslant 2(s-|B|+1)+\operatorname{ord}-\operatorname{match}(G)-2+|B|-1 \\
& \leqslant 2 s-|B|+\operatorname{ord}-\operatorname{match}(G)-1 \\
& \leqslant 2 s+\operatorname{ord}-\operatorname{match}(G)-3 .
\end{aligned}
$$


Case 3. If $|B|=1$, then $B=\left\{x_{1}\right\}$ and $A=\left\{x_{2}, \ldots, x_{d}\right\}$. Therefore, $G \backslash A$ is the disjoint union of $G \backslash N_{G}\left[x_{1}\right]$ and the isolated vertex $x_{1}$. Thus,

$$
I(G \backslash A)^{(s-|B|+1)}=I\left(G \backslash N_{G}\left[x_{1}\right]\right)^{(s)}
$$

and the induction hypothesis implies that

$$
\begin{aligned}
& \operatorname{reg}\left(\frac{S}{I(G \backslash A)^{(s)}: \mathrm{x}_{B}}\right)+|B|-1=\operatorname{reg}\left(\frac{S}{I\left(G \backslash N_{G}\left[x_{1}\right]\right)^{(s)}}\right) \\
& \leqslant 2 s+\operatorname{ord}-\operatorname{match}\left(G \backslash N_{G}\left[x_{1}\right]\right)-2 \leqslant 2 s+\operatorname{ord-match}(G)-3,
\end{aligned}
$$

where the last inequality follows from [19, Lemma 2.1].

Hence, in all cases, we have

$$
\operatorname{reg}\left(\frac{S}{I(G \backslash A)^{(s)}: \mathrm{x}_{B}}\right)+|B|-1 \leqslant 2 s+\operatorname{ord}-\operatorname{match}(G)-3 .
$$

There, using inequality (5), we conclude that

$$
\operatorname{reg}\left(S / I_{1}^{\prime}\right) \leqslant 2 s+\operatorname{ord}-\operatorname{match}(G)-3
$$

and this completes the proof.

As an immediate consequence of Theorem 3, we obtain the following corollary.

Corollary 4. Let $G$ ba chordal graph. Then for any integer $s \geqslant 1$,

$$
\operatorname{reg}\left(I(G)^{(s)}\right) \leqslant 2 s+\operatorname{match}(G)-1 .
$$

The class of Cameron-Walker graphs is an interesting class of graphs, introduced in [6]. It consists of graphs for which ind-match $(G)=\operatorname{match}(G)$. Algebraic properties of Cameron-Walker graphs have been studied in [13, 14] and [21]. The following result follows from Corollary 4 and [9, Theorem 4.6].

Corollary 5. Let $G$ ba chordal Cameron-Walker graph. Then for any integer $s \geqslant 1$,

$$
\operatorname{reg}\left(I(G)^{(s)}\right)=2 s+\text { ind-match }(G)-1 .
$$

The structure of Cameron-Walker graph has been determined in [6]. Indeed, a connected graph $G$ is a Cameron-Walker graph if and only if it is

- a star graph, or

- a star triangle, or

- consisting of a connected bipartite graph $H$ with vertex partition $V(H)=X \cup Y$ such that there is at least one pendant edge attached to each vertex of $X$ and that there may be possibly some pendant triangles attached to each vertex of $Y$.

Thus, Corollary 5 , essentially determines the regularity of symbolic powers of edge ideal of $G$, if either $G$ is a star triangle, or the graph $H$ above is a tree.

Let $n \geqslant 3$ be a positive integer and suppose that $e$ is an edge of the complete graph $K_{n}$. Obviously, the graphs $K_{n}$ and $K_{n}-e$ are chordal graphs and their ordered matching number is one. Thus, by using Theorem 3 and [9, Theorem 4.6], we conclude the following corollary. 
Corollary 6. Assume that either $G=K_{n}$ or $G=K_{n}-e$, for some integer $n \geqslant 3$ and some edge $e \in E\left(K_{n}\right)$. Then for any $s \geqslant 1$, we have

$$
\operatorname{reg}\left(I(G)^{(s)}\right)=2 s .
$$

\section{References}

[1] A. Alilooee, A. Banerjee. Powers of edge ideals of regularity three bipartite graphs. J. Commut. Algebra, 9:441-454, 2017.

[2] A. Alilooee, S. Beyarslan, S. Selvaraja. Regularity of powers of edge ideal of unicyclic graphs. Rocky Mountain J. Math., to appear.

[3] A. Banerjee. The regularity of powers of edge ideals. J. Algebraic Combin., 41:303$321,2015$.

[4] A. Banerjee, S. Beyarslan, H. T. Hà. Regularity of edge ideals and their powers, preprint.

[5] S. Beyarslan, H. T. Hà, T. N. Trung. Regularity of powers of forests and cycles. J. Algebraic Combin., 42:1077-1095, 2015.

[6] K. Cameron, T. Walker. The graphs with maximum induced matching and maximum matchingthe same size. Discrete Math., 299:49-55, 2005.

[7] A. Constantinescu, M. Varbaro. Koszulness, Krull dimension, and other properties of graph-related algebras. J. Algebraic Combin., 34:375-400, 2011.

[8] H. Dao, C. Huneke, J. Schweig. Bounds on the regularity and projective dimension of ideals associated to graphs. J. Algebraic Combin., 38:37-55, 2013.

[9] Y. Gu, H. T. Hà, J. L. O'Rourke, J. W. Skelton. Symbolic powers of edge ideals of graphs, preprint.

[10] H. T. Hà. Regularity of squarefree monomial ideals. In S.M. Copper and S. SatherWagstaff(Ed.) Connections Between Algebra, Combinatorics, and Geometry,, volume 5609 of Springer Proceedings in Mathematics 85 Statistics, pages 251-276. Springer, 2014.

[11] H. T. Hà, A. Van Tuyl. Monomial ideals, edge ideals of hypergraphs, and their graded Betti numbers. J. Algebraic Combin., 27:215-245, 2008.

[12] J. Herzog, T. Hibi. Monomial Ideals, Springer-Verlag, 2011.

[13] T. Hibi, A. Higashitani, K. Kimura, A. B. O'Keefe. Algebraic study on CameronWalker graphs. J. Algebra, 422:257-269, 2015.

[14] T. Hibi, K. Kimura, K. Matsuda, A. Tsuchiya. Regularity and $a$-invariant of Cameron-Walker graphs, preprint.

[15] A. V. Jayanthan, N. Narayanan, S. Selvaraja. Regularity of powers of bipartite graphs. J. Algebraic Combin., 47:17-38, 2018.

[16] M. Katzman. Characteristic-independence of Betti numbers of graph ideals. J. Combin. Theory, Ser. A, 113:435-454, 2006. 
[17] F. Khosh-Ahang, S. Moradi. Regularity and projective dimension of edge ideal of $C_{5}$-free vertex decomposable graphs. Proc. Amer. Math. Soc., 142:1567-157, 2014.

[18] M. Moghimian, S. A. Seyed Fakhari, S. Yassemi. Regularity of powers of edge ideal of whiskered cycles. Comm. Algebra, 45:1246-1259, 2017.

[19] S. A. Seyed Fakhari. Depth, Stanley depth and regularity of ideals associated to graphs. Arch. Math. (Basel), 107:461-471, 2016.

[20] S. A. Seyed Fakhari, S. Yassemi. Improved bounds for the regularity of edge ideals of graphs. Collect. Math., 69:249-262, 2018.

[21] T. N. Trung, Regularity, matchings and Cameron-Walker graphs, preprint.

[22] R. Woodroofe. Matchings, coverings, and Castelnuovo-Mumford regularity. J. Commut. Algebra, 6:287-304, 2014. 